\title{
Thermal analysis and measurements of spiral inductors using built-in and IR sensors
}

\author{
by M. Kałuża*, M. Felczak, and B. Więcek
}

* Institute of Electronics, Lodz University of Technology, Poland, marcin.kaluza@p.lodz.pl

\begin{abstract}
The aim of this paper is to present and compare a contact and a contactless method of rapid thermal characterisation of spiral inductors. A series of spiral inductors of different geometries were fabricated on FR4 PCB and aluminium based MCPCB substrates of different thickness. Next their thermal characteristics were measured using a JESD51-1 compliant contact method implemented in the T3STER tester. The same measurements were repeated using a contactless approach, with a thermographic camera. Next, the results obtained with both methods were compared, showing the advantages and drawbacks of each method.
\end{abstract}

\section{Introduction}

Spiral inductors are widely used for different applications, at different circuit levels, integrated on chip, on PCB or in ceramic packages. Because of their relatively large sizes compared to neighbouring elements, they are becoming a source of non-negligible interferences - both electromagnetic and thermal - especially if used as part of power circuits. Knowing that the principal causes of electronic system failures are namely dust (6\%), humidity (19\%), vibration (20\%) and temperature (55\%) - the last of those factors being clearly dominant - it is obvious that it has to be taken into account in the design process. Because of specific spiral inductors geometry, whit three to four orders off magnitude difference when comparing their thickness to external length or width, spiral simulations are numerically ill-conditioned and very time consuming, especially if full 3D calculations are required. One of the solutions of this problem consist in rapid prototyping and direct thermal characterization of structures containing spirals, based on their thermal response measurements.

A generally accepted in the electronic industry reference for the thermal measurements and characterization of integrated circuits and packages is the series of JEDEC JESD51 [1] standards, and especially the JESD51-1 standard "Integrated Circuit Thermal Measurement Method - Electrical Test Method (Single Semiconductor Device) [2], which defines a standard Electrical Test Method (ETM) that can be used to thermally characterize single integrated circuit devices housed in electric packages or to compare such circuits or packages.

To characterize electronic circuits, the JESD51-1 standard uses the concept of thermal resistance defined as:

$$
R_{\theta J X}=\frac{T_{J}-T_{X}}{P_{H}}
$$

where $\quad R_{\theta J X}$ is the thermal resistance between the device junction and the specific environment.

$T_{J}$ is the junction temperature

$T_{X}$ is the environment reference temperature

$P_{H}$ is power dissipated in the device

which describes the thermal resistance between the junction (heat source) and a reference point, which could, for example, be the ambient temperature, case temperature or board temperature. The thermal resistance, given in K/W, is a measure of heat removal capability.

The standard proposes two different methods of measurement. The first one called Dynamic Mode is based on switching between heating with pulses of specific duration and temperature measurement. It is also called the Pulsed Mode. The second approach, called Static Method, consist in applying a unit power step to the device under test and to continuously monitor its temperature, thus it requires only one switching and eliminates the problem of pulse duration adaptation.

Thermal resistance is a steady state parameter, and thus can not - in general - capture the dynamic behaviour of tested circuits. A convenient parameter for dynamic characterization of the devices is the thermal impedance $Z_{\text {th }}$ [3- 5]. Using the same methods as described in the JESD51-1 standard, one can measure the transient thermal response of the device under test to a heating or cooling step function $T_{\text {step }}(t)$. The time domain characteristic can next be transformed into frequency domain using Fourier transform, giving finally the circuit complex thermal impedance versus frequency, which can be presented in the form of Nyquist plot. The complex thermal impedance can be calculated using the equation (2). 


$$
Z_{\text {th }}(j \omega)=\frac{T(j \omega)}{P(j \omega)}=\frac{T_{\text {step }}(j \omega)}{P_{0} F\{1(t)\}}=\frac{j \omega}{P_{0}} T_{\text {step }}(j \omega)=\frac{j \omega}{P_{0}} \int_{0}^{\infty} T_{\text {step }}(t) e^{-j \omega t} d t
$$

where $F$ denotes Fourier transform, $T_{\text {step }}$ is the temperature response to a heating or cooling step function, $\omega$ is the angle frequency and $P_{0}$ is the power delivered to the inductor in steady state.

\section{Measurement setup}

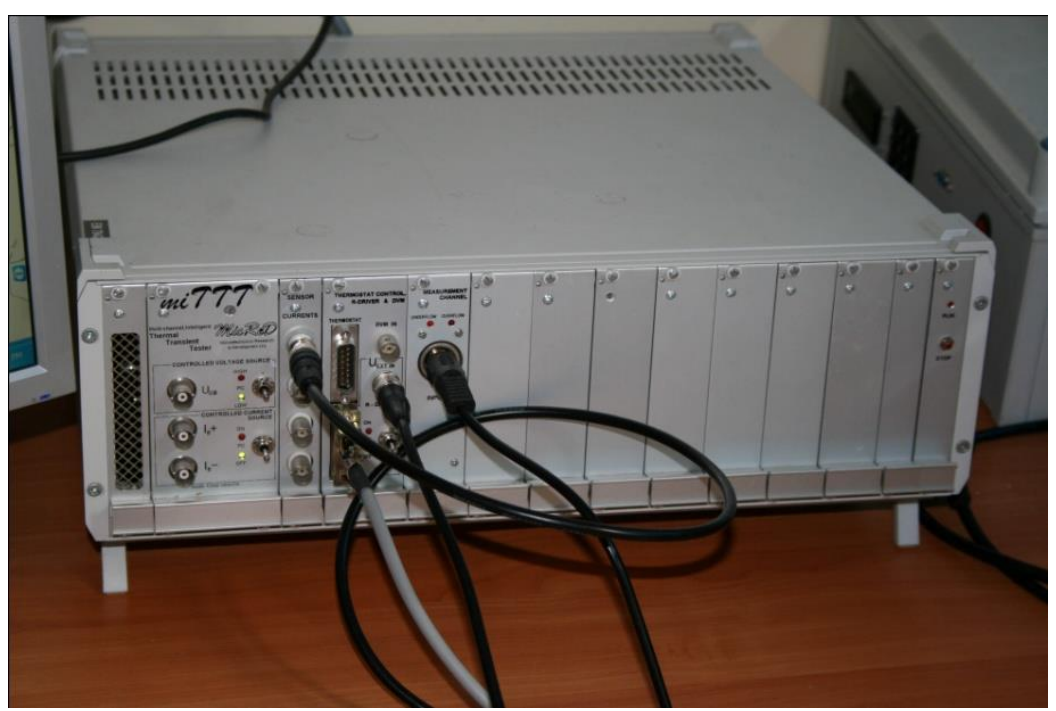

Fig. 1. T3STER main system unit.

For the purpose of the presented work two different measurement setups were used. The first one was the T3STER - Transient Thermal Tester by MICRED/Mentor Graphics, which is one of the references in the electronics industry for circuits thermal design and management (fig. 1). The system was composed of the main system hardware unit and of dedicated software for measurement results analysis. A block schematic of the tester is shown in fig. 2 . The unit consists of the power channel, which is responsible for powering and heating/cooling the device under test (in some cases an external power source controlled by an internal power switch can be used to overcome voltage or current limits). The second part of the tester is the measurement unit, containing controlled voltage and current sources and A/D converters. Those two modules are controlled by a common digital control unit.

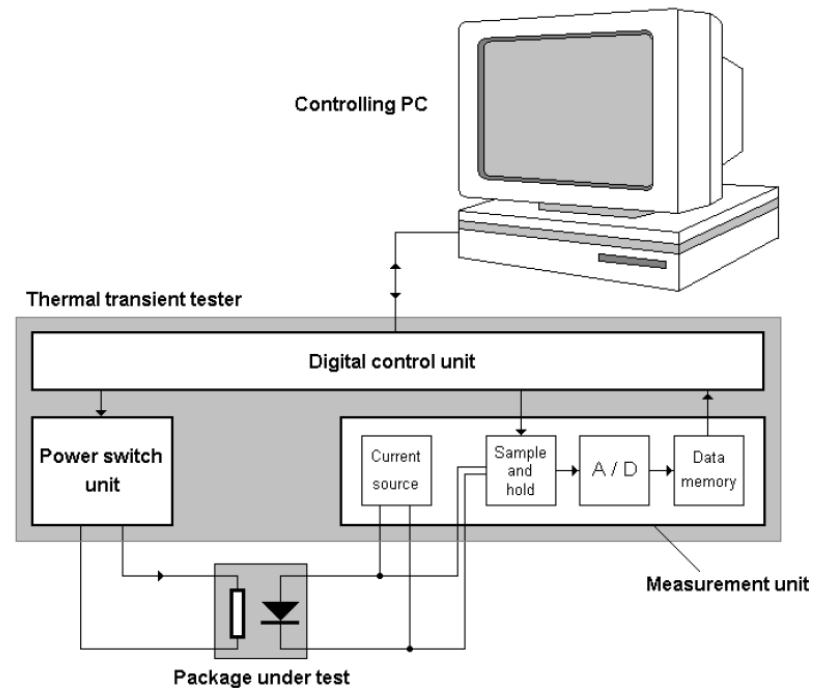

Fig. 2. Block schematic of the thermal transient tester hardware [6].

The T3STER unit uses the JEDEC JESD51-1 static measurement mode, but instead of the steady-state measurements $\left(R_{\theta J x}\right)$, offers dynamic modelling of the tested circuits in the form of pulsed thermal resistance, thermal impedance, complex impedance Nyquist plot, time constant spectrum or structural functions [6]. In the setup used for measurements, 4 different sensor configurations were available (fig. 3): a MOSFET transistor, a bipolar transistor, a 
diode in which the sensing element was also used as power dissipator. The fourth configuration allowed to use a resistive load as heat source in combination with a semiconductor diode as sensing element. The sensor configuration choice was based on their inherent feature of sensing temperature, with $U_{B E}(B J T), U_{G S}(M O S)$ or $I_{F}$ (diode) parameters influenced by the temperature (fig. 4).

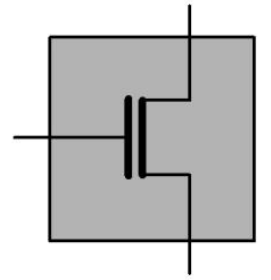

a)

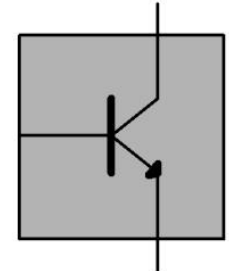

b)

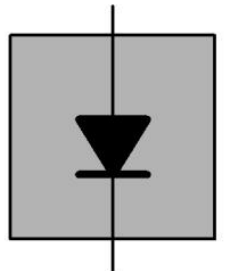

c)

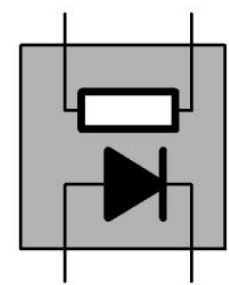

d)

Fig. 3. Available T3STER sensor configurations [6].

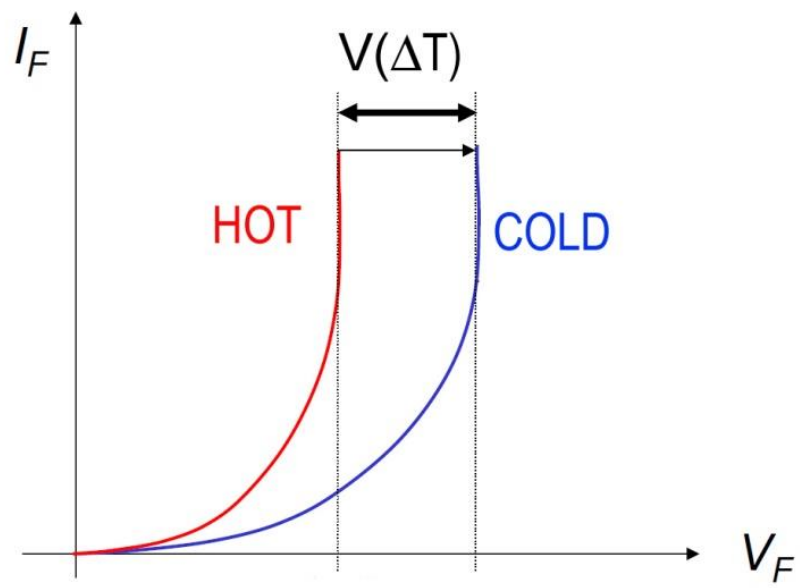

Fig. 4. Principle of operation of T3STER configuration using a diode as temperature sensor.

Because of the circuits used for measurements, i.e. spiral inductors of different geometries fabricated on FR4 PCB and aluminium based MCPCB substrates of different thickness, the diode-resistor configuration was in this case an obvious choice for measurements.

The second measurement setup used for the purpose of the presented work was a MWIR Cedip Titanium camera with a cooled InSb $640 \times 512$ pixels detector matrix ( $15 \mu \mathrm{m}$ pixel pitch) and a $20 \mathrm{mK}$ NETD value. To allow fast and easy camera positioning, the camera was mounted on a vertical stand.

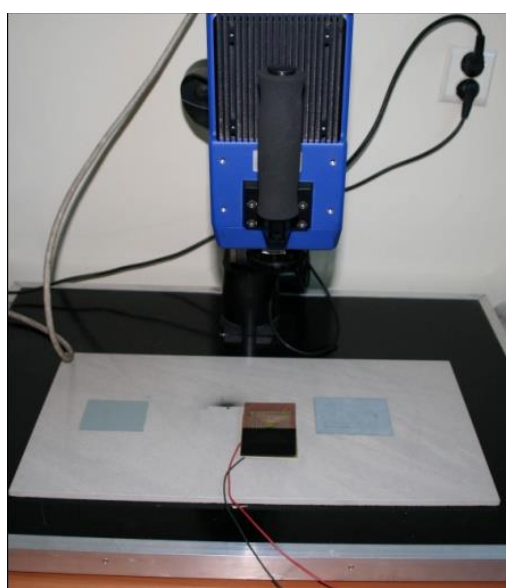

Fig. 5. Thermographic measurement stand with MWIR Cedip Titanum camera mounted on. 


\section{Measurement results}

A series of spiral inductors of 3 different geometries were fabricated on FR4 PCB and aluminium based MCPCB substrates of different thickness. They were 4 different FR4 substrates: 0.5, 1.0, 1.5 and $2.0 \mu \mathrm{m}$ thick and two different aluminium MCPCB substrates: 1.0 and $1.5 \mu \mathrm{m}$ thick. In all cases the copper layer had a thickness of $35 \mu \mathrm{m}$. It gave a total of 18 different test circuits. For the purpose of this article are presented the measurement results for a 6 turn, $50 \times 50 \mathrm{~mm}$ test spiral on $1.5 \mu \mathrm{m}$ thick FR4 PCB (further down test spiral no 1) and $1.5 \mu \mathrm{m}$ thick МСРСB aluminium substrate, with a $0.1 \mathrm{~mm}$ thick dielectric layer between aluminium and copper (further down test spiral no 2). Both spirals had the same geometry: trace width - $1 \mathrm{~mm}$, edge-to-edge segments distance - $1 \mathrm{~mm}$, copper thickness $38 \mu \mathrm{m}$, and the substrate external dimensions of $10 \times 7 \mathrm{~cm}$ (fig. 6). The spirals were heated using a power step function.

For the T3STER measurements, as described in the previous section, the resistance-diode configuration was used, with the diode used as temperature sensor applied directly on the spiral metallization surface ( $3^{\text {rd }}$ turn). The value of the heating current was limited to $2 \mathrm{~A}$ by the T3SETR internal power switch unit.

In order to achieve consistent and comparable results, T3STER and thermographic measurements were done simultaneously, with the camera placed above the device under test and recording the temperature changes during T3STER measurements.

Additionally, to solve the problem of low copper emissivity, half of each spiral was painted using black mate paint (fig. 5). Numerical results of those measurements were extracted from thermograms and next converted using a purpose written Matlab program to a suitable format, compatible with internal T3STER format, thus allowing for analyzing thermographic contactless results in the tester dedicated software.

The obtained results are presented in fig. 7 to 10 below, whereas the final conclusions based on those results are presented in the next section.

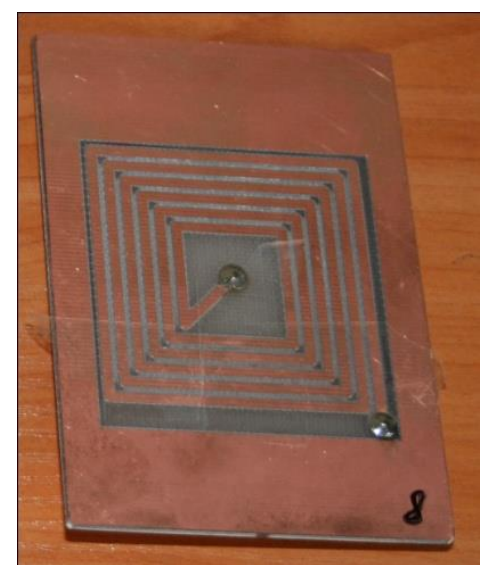

Fig. 6. 6-turn, $50 \times 50 \mathrm{~mm}$ test spiral on $1.5 \mu \mathrm{m}$ thick FR4 PCB (trace width - $1 \mathrm{~mm}$, edge-to-edge segments distance - $1 \mathrm{~mm}$, copper thickness $38 \mu \mathrm{m})$.

In fig. 7 are presented the results of cooling process temperature measurement for spiral no 1 , for T3STER contact (on the left) and thermographic contactless method. Those results were the basis for further analysis: circuit time constant spectrum, dynamic pulse thermal resistance and complex thermal resistance calculation. As shown in fig. 7, for contact measurements a strong temperature overshot and undershot was observed during first microseconds, whereas for longer times both methods were giving similar results. Because of this problem only thermographic data were used for further analysis. An explanation of this phenomena will be given in the conclusions. 

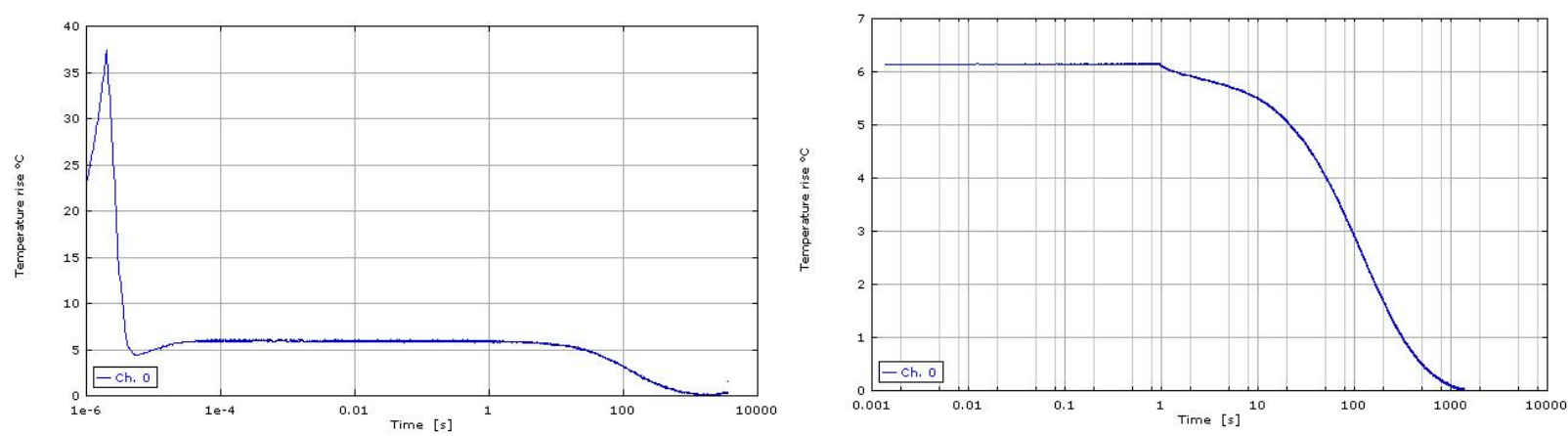

Fig. 7. Cooling curve for the spiral no 1: T3STER on the left, thermography on the right. A strong temperature overshot and undershot visible in the contact measurement curve during first microseconds.

Based on cooling process temperature measurements, the complex thermal impedance was calculated. The results in the form of Nyquist plot are shown in fig. 8 for spiral no 1 (FR4 - left) and spiral no 2 (MCPCB - right). As one can see, for low frequency there is a clear difference in the real part of impedance value between both circuits (around $9 \mathrm{KM}$ compared to $16 \mathrm{~K} / \mathrm{W}$ respectively), demonstrating a much better heat dissipation capability of aluminium vs epoxy substrate. On fig. 9 are presented the pulse thermal resistance curves for spiral no 1 (left) and spiral no 2 ( right). The pulse thermal resistance diagram describes the behaviour of the device when driven by repeated pulses of given length and duty cycle (PWM like modulation), with time on the $X$ axis and the effective thermal resistance on the $Y$ axis. As one can see, for long heating pulses and high waveform fill factor the value of the dynamic thermal resistance is reaching the static thermal resistance value. Analysing those results one can see again that the aluminium based substrate shows considerably better cooling properties than the epoxy one (around $9 \mathrm{KM}$ compared to $16 \mathrm{~K} / \mathrm{W}$ respectively). Moreover, the pulse thermal resistance curves are allowing to identify the structure of both circuits: a 2 layer in the case of FR4 PCB and a 3 layer in the MCPCB case.
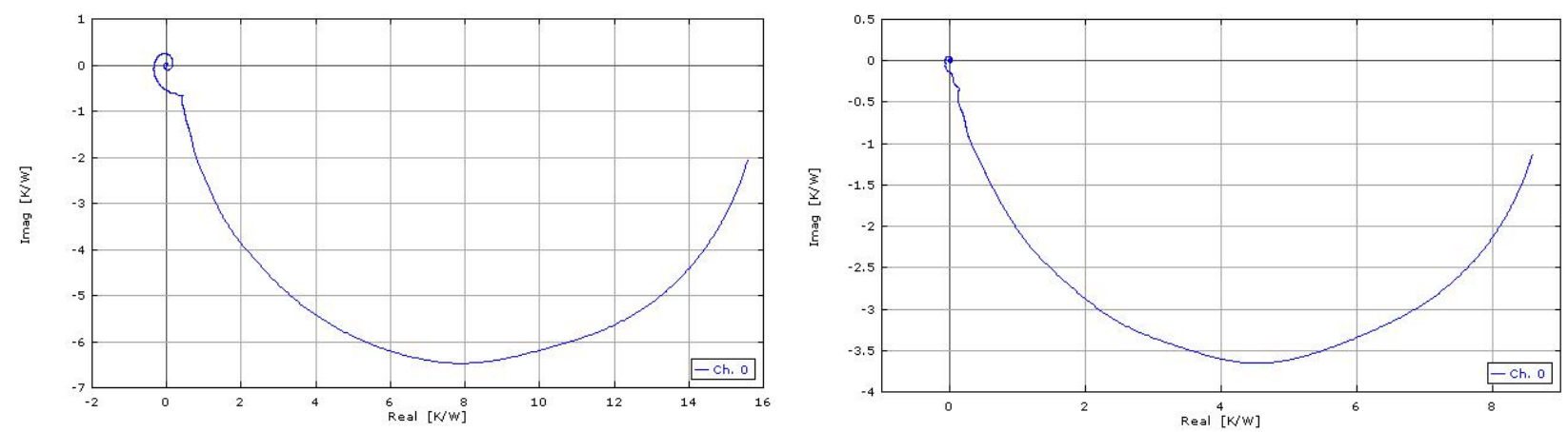

Fig. 8. Complex thermal resistance curves calculated for spiral no 1 (left) and spiral no 2 (right), based on cooling process thermographic measurements.
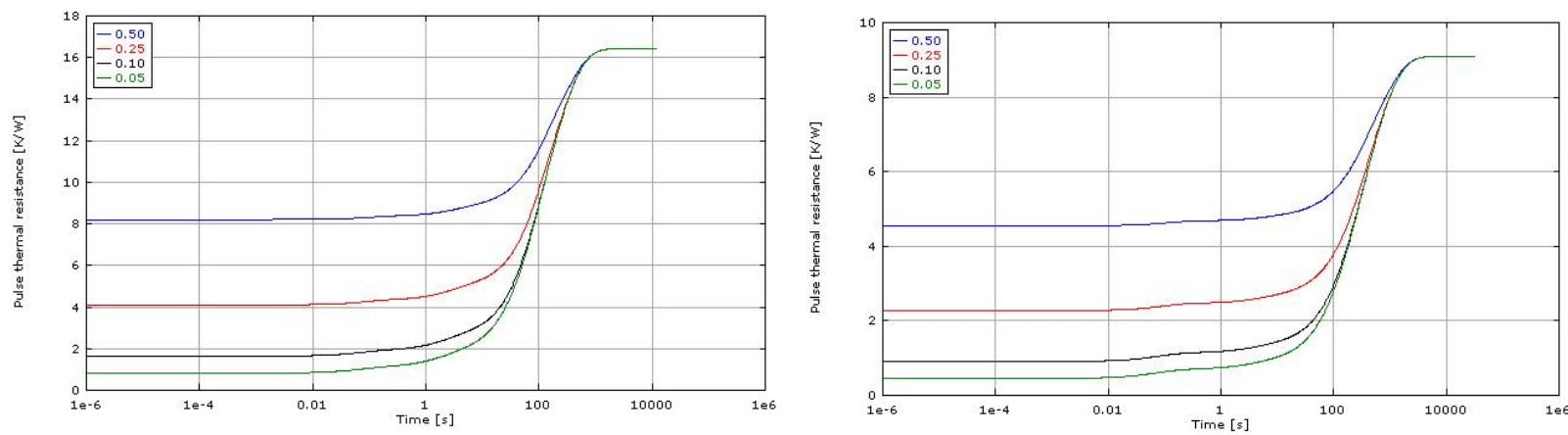

Fig. 9. Pulse thermal resistance curves calculated for spiral no 1 (left) and spiral no 2 (right), obtained based on cooling process thermographic measurements. 
The T3STER system allows also to present the thermal characteristics of the device under test in the form of so called "tau intensity diagram" [7], which is a circuit thermal time constant spectrum diagram. This representation of the circuit allows to clearly identify its structure, the number of time constants being equivalent to the number of layers in the heat conduction chain and to demonstrate how fast the heat is dissipated, from source to the environment (final layer). The tau intensity diagram for spiral no 1 (left) and spiral no 2 (right) based again on thermographic measurements - are shown on figure 10.
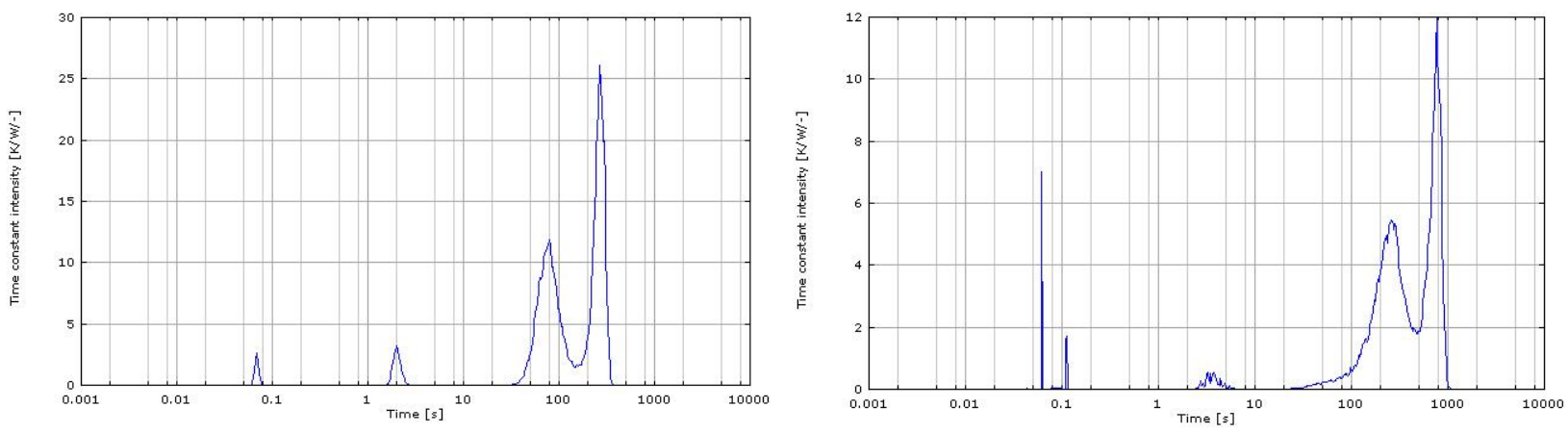

Fig. 10. Tested circuit tau intensity diagram (time constant spectrum) for spiral no 1 (left) and spiral no 2 (right), obtained based on cooling process thermographic measurements.

\section{Conclusions}

The aim of this paper was to present and compare two different methods of rapid thermal characterisation of spiral inductors: a contact one, using the Transient Thermal Tester (T3STER) system and a contactless one, based on thermographic measurements. For the same spiral inductor geometry fabricated on PCB and aluminium MCPCB substrate, the temperature during the circuit cooling process was measured using a silicon diode and a thermal camera. The data from the camera, after conversion, was feed to the T3STER system and compared to the contact measurement results. As was shown in figure 7, there was a considerable difference between results obtained using both methods. Further investigations showed that thermographic measurements were repeatable, whereas repeating contact measurements with diode as temperature sensor was giving inconsistent results. The difference of results observed came from a combination of several factors. The circuit under test was an inductance, thus one should expect some oscillations during power switching on or off. Those oscillations are clearly visible in figure 7 (left). Because T3STER sampling frequency, as given in the system manual, is $1 \mathrm{MHz}$ and the camera frame rate was much lower, at 720 fps, those fast transient phenomena were just filtered out when using thermography. The second source of the problem was the inductive coupling between the spiral inductor and the diode leads and connection wires, consisting and additional source of error. This was eliminated when using contactless approach. The last element was the efficiency of heat transfer from the circuit to the sensor diode (i.e. the quality of thermal contact), which also had an influence on the contact measurement results.

The version of the T3Ster software used for the analysis allowed to filter and smooth the first 1 up to $1000 \mu \mathrm{s}$ of the cooling curve, however in the case of tested inductors the results were not satisfactionary. Thus, finally, to prove the role of fast transient oscillations and inductive coupling, the cooling temperature curve measured with a diode as sensor was smoothed manually for the first $1 \mathrm{~ms}$ and then feed to the analysis program. The results obtained in this case were close to the one obtained based on thermography (figure 11).
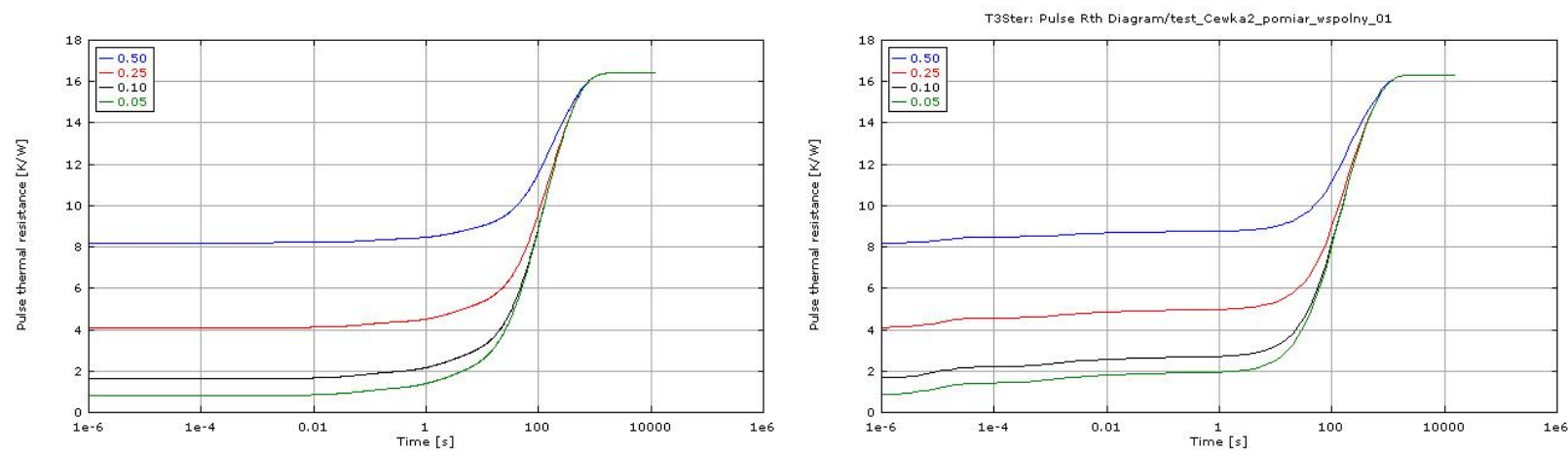

Fig. 11. Pulse thermal resistance curves calculated for spiral no 1: left - thermography, right - contact measurement results smoothed manually. 
When applied to thermal characterization of spiral inductors both methods showed their strength and weaknesses. The advantage of the contact method is its sampling speed, three orders of magnitude greater than the one obtained with the IR camera used for measurements. The drawback of this method is the quality of thermal contact between the sensor and the circuit under test (can be eliminated if using sensors integrated in the circuit, which is not always an available option) and the problem of coupling if measuring inductive circuits. Thermography offers immunity to EMC related problems, lack of influence of measurement circuit on the tested device but at the cost of much lower acquisition speed and problems if the surface of the device under test is a low emissivity one. For the spiral inductor circuits tested, given their sizes, as demonstrated, the camera measurement speed was not an issue. In this case the contactless, thermographic approach was found to be more straightforward, giving better results, with more confidence.

\section{REFERENCES}

[1] JEDEC JESD51 Methodology for Thermal Measurement of Component Packages (SIngle Semiconductor Device)

[2] JEDEC JESD51-1 Integrated Circuit Thermal Measurement Method - Electrical Test Method (Single Semiconductor Device)

[3] P. Kawka, G. De Mey, B. Vermeersch, "Thermal characterization of electronic packages using the Nyquist plot of the thermal impedance", IEEE Transaction on Components and Packaging Technologies, 2007, vol.30, pp. 660665.

[4] B. Vermeersch, G. De Mey, "Thermal impedance plots of micro scaled devices", Microelectronics Reliability, 2006, vol.46, pp.174-177.

[5] B. Vermeersch, G. De Mey, "Influence of substrate thickness on thermal impedance of microelectronic structures", Microelectronics Reliability, 2007, vol.47, pp.437-443.

[6] T3STER - Transient Thermal Tester software manual

[7] V. Székely, M. Rencz: "Thermal dynamics and the time constant domain", IEEE Transactions on Components and Packaging Technologies, V.23, No.3. September 2000, pp. 587-594 